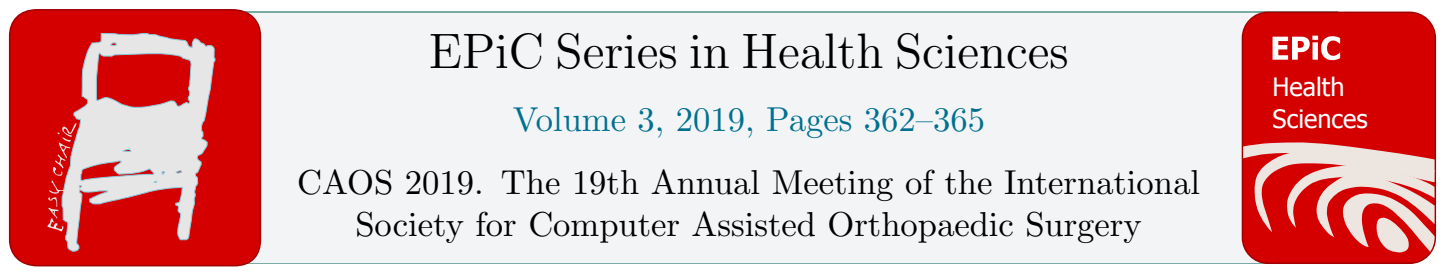

\title{
Femoral component rotation angle after unicompartmental knee arthroplasty using the anatomical tibial component cutting technique with computer-assisted surgery by CT-scan measurement in Rajavithi hospital
}

\author{
Kittikun Thienthong, MD Pornpavit Sriphirom, MD Anuchit Vejjaijiva, MD Soros \\ Suwansawaiphol, MD Rajavithi Hospital , Rangsit university \\ ${ }^{1}$ Rajavithi Hospital, Rangsit University, Bangkok, Thailand \\ ${ }^{2}$ Ranong Hospital, Ranong, Thailand \\ ${ }^{3}$ Banphaeo Hospital Sathorn Branch, Bangkok, Thailand \\ ${ }^{4}$ Medical Development Clinic, Bangkok, Thailand \\ pornpavit@yahoo.com, Golf342000@yahoo.com, chaisira@hotmail.com, \\ b_chanopas@yahoo.com
}

\begin{abstract}
Complication following UKA in medial compartment OA knee often related to rotatory malposition of the femoral component The purpose of this study was to study outcome following Femoral component rotation angle after unicompartmental knee arthroplasty using the anatomical tibial component cutting technique with computer-assisted surgery by CT-scan measurement in Rajavithi hospital

Retrospective descriptive study of 16 patients performed medialUKA in Rajavithi Hospital Bangkok Thailand.Single orthopedic surgeon and using computer-assisted with anatomical tibia cutting technique(tibial resection in 3-degree varus).

The femoral component rotation was assessed in an axial cut $\mathrm{CT}$ scan. measure angle between Epicondylar axis and Posterior condylar axis

The authors analyzed 16 knees with mean age about 56 years, male 2 Female 14 and the mean BMI was $24 \mathrm{~kg} / \mathrm{m} 2$ (average body weight $63 \mathrm{~kg}$ )

Mean of Femoral component rotation angle in this study is 2.94-degree internal rotation(internal rotation compare with Epicondylar axis). A range of femoral component internal rotation was 0-6 degree .mean varus-valgus angle was 1.81-degree varus and mean flexion-extension angle was 5.56-degree flexion

Femoral component rotation angle in unicompartmental knee arthroplasty(UKA) in medial compartment osteoarthritis knee with using of the computer-assisted with anatomical tibia cutting
\end{abstract}


Femoral component rotation angle after unicompartmental knee arthroplasty ... K. Thienthong et al.

technique have a favorable result $($ Mean $=2.94$-degree internal rotation 1.81-degree varus and 5.56degree flexion).

\section{Introduction}

Unicompartmental knee arthroplasty (UKA) has been performed for the treatment of medial or lateral femorotibial knee osteoarthritis for more than three decades. The long-term clinical outcomes of UKA depend on patient selection, age, sex, and level of activities ${ }^{[1][2]}$

UKA has demonstrated several benefits, including shorter postoperative recovery ${ }^{[3]}$, improved knee range of motion ${ }^{[4]}$, and preservation of near-normal knee kinematics ${ }^{[5]}$.

UKA has also been shown to have a relatively low morbidity rate with less blood loss and low rates of complications ${ }^{[6-8]}$. however, the higher the risk of revision after UKA compared to TKA has also been reported ${ }^{[9]}$

Component malposition correlated with suboptimal point contact and increased stress concentration have been identified as main contributors for accelerated polyethylene wear, poor functional outcomes, and increased revision rate. In order to obtain a satisfactory outcome after UKA, proper surgical technique and optimal implant positioning are necessary.

Complication following unicompartmental knee arthroplasty(UKA) in medial compartment osteoarthritis knee often related to rotatory malposition of the femoral component ${ }^{[10]}$.

The purpose of this study was to study outcome following Femoral component rotation angle after unicompartmental knee arthroplasty using the anatomical tibial component cutting technique with computer-assisted surgery by CT-scan measurement in Rajavithi hospital.

\section{Materials and Methods}

Retrospectively, the medical and radiographic data of all patients who underwent a medial UKA using the CAS fixed bearing UKA (Orthopilot UKA v.3.0,B.Braun, Aesculap, Tuttlingen, Germany) with anatomical tibia cutting technique were reviewed. Indications for medial UKA in the current study were medial osteoarthritis knee. Postoperative CT was performed to identify femoral component alignment in 3 planes(coronal, sagittal and axial plane) From May 2014 to June 2018, 16 knees met these criteria. Medical records, including sex, age, body mass index, operated side, and tibial and femoral component size, were recorded.

\section{Surgical procedure}

A minimally invasive approach without a release of the superficial medial collateral ligament and adjacent tendinous structures was utilized. Following removal of the medial osteophytes, After tracker fixation, landmark registration, the range of movement and laxity mapping, the anatomy of the femoral condyle and the tibial plateau was mapped. Planning for component size, alignment and position, and the volume of bone removal is determined intraoperatively by the surgeon. After registration, the tibial extramedullary cutting guides were attached and adjusted to match the coronal and sagittal alignments. Then, a sagittal cut of the tibia was performed along a line parallel to Akagi's line, in the most lateral rim of the medial plateau. The femoral distal cutting block was also adjusted to match the coronal and sagittal alignments by using navigation. When the femoral posterior bone cut was performed, the femoral rotational angle was aligned. We recorded the femoral and tibial boneresection angles with respect to the mechanical axis intraoperatively by using the navigation system. 
Femoral component rotation angle after unicompartmental knee arthroplasty ... K. Thienthong et al.

\section{Radiological evaluation}

Postoperative CT scan was obtained at 2 weeks after the operation. Radiological measurements were performed twice by the 2 observers.

Rotation of the femoral component was measured using two reference lines between Epicondylar Axis and Posterior condylar Axis. Sagittal rotation (flexion/extension) was measured from a sagittal femoral mechanical axis and Frontal rotation (varus/valgus) was measured from the mechanical axis.

\section{Results}

The authors analyzed 16 knees with mean age about 56 years, male 2 Female 14 and the mean BMI was $24 \mathrm{~kg} / \mathrm{m} 2$ (average body weight $63 \mathrm{~kg}$ )

Mean of Femoral component rotation angle in this study is 2.94-degree internal rotation(internal rotation compare with Epicondylar axis). A range of femoral component internal rotation was $0-6$ degree .mean varus-valgus angle was 1.81-degree varus and mean flexion-extension angle was 5.56-degree flexion

\section{Discussion}

The most important finding of this study was the femoral component rotation with using the CAS fixed bearing UKA in anatomical tibia cutting technique. Kyoung-Tak Kang et a ${ }^{[11]}$ showed that Femoral component position could be one of the sensitive factors that influenced the contact stresses on the PE insert and articular cartilage, and the postoperative significance of the femoral component position in UKA. Assor $\mathrm{M}$ et $\mathrm{a}^{[12]}$ found that Rotatory malposition of the femoral component of a unicompartmental knee arthroplasty (UKA) is

a key element of mechanical failure. But both of study focus on the position of a femoral component and frontal and sagittal plane of the femoral component. In our study focus on femoral rotational alignment. from our study, we performed CAS UKA to recreate a nearly native joint line by selected angle of tibial bone cut according to native mechanical tibial alignment. Our study found that Mean of Femoral component rotation angle in this study is 2.94-degree internal rotation mean varus-valgus angle was 1.81-degree varus and mean flexion-extension angle was 5.56-degree flexion. Even so, this study demonstrated that using of the computer-assisted with anatomical tibia cutting technique have a favorable result.

There were some limitations of this study that the author had concerned. First, the study population was only sixteenth patients. second limitation is that this study is retrospective. Nevertheless, this study reports results using of the computer-assisted with anatomical tibia cutting technique in UKA. However, this study needs to increase the number of patients and need more clinical data to support this study. 
Femoral component rotation angle after unicompartmental knee arthroplasty ... K. Thienthong et al.

\section{Conclusion}

Femoral component rotation angle in unicompartmental knee arthroplasty(UKA) in medial compartment osteoarthritis knee with using of the computer-assisted with anatomical tibia cutting technique have a favorable result (Mean $=2.94$-degree internal rotation 1.81-degree varus and 5.56degree flexion).

\section{Reference}

1. Jung KA, Kim SJ, Lee SC, Hwang SH, Ahn NK. Accuracy of implantation during computerassisted minimally invasive Oxford unicompartmental knee arthroplasty: a comparison with a conventional instrumented technique. Knee. 2010;17:387e391.

2. Keene G, Simpson D, Kalairajah Y. Limb alignment in computer-assisted minimally-invasive unicompartmental knee replacement. J Bone Joint Surg Br. 2006;88:44e48.

3. Lombardi $\mathrm{Jr} \mathrm{AV}$, Berend $\mathrm{KR}$, Walter $\mathrm{CA}$, et al. Is recovery faster for mobilebearing unicompartmental than total knee arthroplasty? Clin Orthop Relat Res 2009;467(6):1450

4. Laurencin CT, Zelicof SB, Scott RD, et al. Unicompartmental versus total knee arthroplasty in the same patient. A comparative study. Clin Orthop Relat Res 1991;273(12):151.

5. Patil S, Colwell Jr CW, Ezzet KA, et al. Can normal knee kinematics be restored with unicompartmental knee replacement? J Bone Joint Surg Am 2005;87(2):332.

6. Price AJ, Webb J, Topf H, et al. Rapid recovery after Oxford unicompartmental arthroplasty through a short incision. J Arthroplasty 2001;16(8):970.

7. Peersman G, Stuyts B, Vandenlangenbergh T, et al. Fixed- versus mobile-bearing UKA: a systematic review and meta-analysis. Knee Surg Sports Traumatol Arthrosc 2014;23(11):3296.

8. Liddle AD, Pandit H, Judge A, et al. Patient-reported outcomes after total and unicompartmental knee arthroplasty: a study of 14,076 matched patients from the National Joint Registry for England and Wales. Bone Joint J 2015;97-B(6):793.

9. Niinimaki T, Eskelinen A, Makela K, et al. Unicompartmental knee arthroplasty survivorship is lower than TKA survivorship: a 27-year Finnish registry study. Clin Orthop Relat Res 2014;472(5):1496.

10. $\underline{\text { Hughes } \mathrm{AJ}^{1}}, \underline{\mathrm{O}^{\prime} \text { hEireamhoin } \mathrm{S}^{1}}, \underline{\underline{\text { Heffernan }}^{2}}, \underline{\text { Hurson } \mathrm{C}^{1}}$. A Simple Approach to Assessment of a Total Knee Replacement's Rotationary Profile Using Computed Tomography. Orthop Surg. 2015 Nov;7(4):350-3. doi: 10.1111/os.12211.

11. Kyoung-Tak Kang, Juhyun Son, Yong-Gon Koh, Oh-Ryong Kwon, Sae Kwang Kwon, Yong Jun Lee, Kwan Kyu Park. Effect of femoral component position on biomechanical outcomes of unicompartmental knee arthroplasty. The Knee 25 (2018) 491-498 